\title{
Younger age at the time of first metastasis in BRAF-mutated compared to BRAF wild-type melanoma patients
}

\author{
MELANIE SAINT-JEAN ${ }^{1,2}$, GAËLLE QUEREUX ${ }^{1,2}$, JEAN-MICHEL NGUYEN $^{3}$, \\ LUCIE PEUVREL $^{1,2}$, ANABELLE BROCARD ${ }^{1,2}$, AUDREY VALLEE ${ }^{4}$, ANNE-CHANTAL KNOL ${ }^{2,5}$, \\ AMIR KHAMMARI ${ }^{2,5}$, MARC G. DENIS ${ }^{4}$ and BRIGITTE DRÉNO ${ }^{1,2,5}$ \\ ${ }^{1}$ Department of Dermato-Cancerology, University Hospital Hôtel-Dieu, Nantes; ${ }^{2}$ Inserm U892, CIC Biothérapie \\ Inserm 0503, Nantes; ${ }^{3}$ PIMESP, Saint-Jacques Hospital, Nantes; ${ }^{4}$ Department of Biochemistry, University Hospital \\ Hôtel-Dieu, Nantes; ${ }^{5}$ Immuno-Dermatology Laboratory, University Hospital Hôtel-Dieu, Nantes, France
}

Received February 5, 2014; Accepted March 26, 2014

DOI: 10.3892/or.2014.3265

\begin{abstract}
The relationship between BRAF mutations and the patient clinical profile is still under question. The objective of the present study was to correlate the BRAF mutation status in primary and metastatic melanomas with the clinicopathological profile, disease-free (DFS) and overall survival (OS). A total of 367 melanoma samples from 278 patients were screened for their BRAF status using a combination of allele-specific amplification and DNA sequencing. Two or three tissue samples from the same patient were available for 74 patients. The clinicopathological characteristics were tested for their association with the BRAF mutation using the Fisher's or Pearson's $\chi^{2}$ test. Log-rank tests and Cox models were used for survival analyses. BRAF mutation was found in 152 samples (41.4\%). Ten of the 74 patients with several tissue samples (13.5\%) had discordant BRAF mutation results. BRAF-mutated patients were significantly younger at the time of primary melanoma and first diagnosis of metastasis than BRAF wild-type patients but with no difference in DFS and OS. According to our results, a primary melanoma with BRAF mutation is not associated with a more aggressive illness.
\end{abstract}

\section{Introduction}

The MAP kinase pathway has been known for a long time to be involved in mediating cell proliferation in response to growth signals. However, BRAF mutations were identified in

Correspondence to: Professor Brigitte Dréno, Department of Dermato-Cancerology, CHU Hôtel-Dieu, 1 Place Alexis Ricordeau, 44093 Nantes Cedex 1, France

E-mail: brigitte.dreno@wanadoo.fr

Abbreviations: DFS, disease-free survival; OS, overall survival; DFI, disease-free interval

Key words: BRAF mutation, disease-free survival, melanoma, overall survival melanoma cell lines and primary tumour samples only 10 years ago using a genome-wide screening method (1). More than 75 somatic BRAF mutations have been described in melanoma (2). Most of these mutations cause uncontrolled activation of the RAF kinase and of the downstream pathway, resulting in cell proliferation and survival.

This discovery has enhanced the research in developing effective target therapies for metastatic melanoma. In a phase III trial comparing vemurafenib to dacarbazine in 675 patients with metastatic melanoma previously untreated, the authors showed an improvement in overall survival (OS) and progression-free survival (PFS) in patients treated with the BRAF inhibitor (3). More recently, another BRAF inhibitor, dabrafenib, has also shown promising results for metastatic melanoma including patients with brain metastases $(4,5)$. The combined treatment of dabrafenib with a MEK inhibitor (trametinib) improves the PFS when compared to dabrafenib alone (6).

However, the relationship between BRAF mutations and the patient clinical profile is still under question. In a metaanalysis published in 2011, the occurrence of the BRAF mutation was associated with a histological superficial spreading melanoma (SSM) subtype and was significantly higher in melanomas located on the trunk than on the face or scalp (7). In another cohort study, a truncal or lower extremity location of the melanoma, younger age and low solar elastosis were independently associated with BRAF mutations (8). A recent retrospective study showed that BRAF mutation correlated with younger age, a higher number of melanocytic naevi and a melanoma location in intermittent UV-exposed skin (9). The link between the BRAF mutation and a poorer outcome remains unclear with contradictory results (10-15).

The aims of the present study were to define the clinical profile of BRAF-mutated patients and to determine the correlation between the BRAF mutation and survival.

\section{Patients and methods}

Patient selection. All consecutive inpatients with melanoma [American Joint Committee on Cancer (AJCC) stage I, II, III or IV (16)] seen in our Unit between January 2011 and 
June 2012 were included. Mucous and ocular melanomas were not excluded. Considering that melanoma is a cancer with a high risk of recurrence, BRAF screening was systematically performed in the primary melanoma, if available, and in the metastases at the Department of Biochemistry. Informed consent was obtained from the study participants who allowed access to their data for scientific objectives, with approval by the local ethics committees. This cohort study focused on clinical correlations and survival in the whole population. Data concerning a part of this series (i.e. the 74 patients with multiple BRAF testing) have been published elsewhere (17).

Data concerning patients were retrospectively collected from clinical notes and the local database in regards to gender, age, and for the primary melanoma: location, histopathological subtype, Breslow thickness, ulceration; for lymph node melanoma: type of sample (biopsy or lymph node excision), number of invaded lymph nodes and capsular breaking; for all melanomas: date of primary melanoma, date and location of progression, treatments received, date of death. For patients with unknown primary melanoma, the date of diagnosis was defined as the date of the first biopsy confirming the presence of melanoma cells. Concerning the location of the primary melanoma, two groups were defined: the 'sun-exposed' location group (including face or scalp, hands, forearms and legs) and the 'non-sun-exposed' location group (including trunk, arms, thighs and feet). Progression of the disease was defined as a change in melanoma stage, i.e. for primary melanoma (stage I or II), appearance of a lymph node, cutaneous or visceral metastasis and for stage III melanoma, recurrence of lymph node or cutaneous metastasis or appearance of visceral metastasis (stage IV). As the present study did not aim to consider the therapeutic effects of the treatments, the progression of stage IV patients as recently updated by the RECIST (Response Evaluation Criteria in Solid Tumors) working group was not considered (18). The OS was defined from the date of primary melanoma diagnosis to the date of death.

Tumour samples. Serial sections were cut from each paraffin block and placed on glass slides. The first section was stained for histopathological examination. The 2-5 subsequent sections were processed for DNA extraction. To enrich the analysed specimen with tumour cells, tumour areas highlighted by a pathologist were macrodissected.

DNA extraction. DNA was extracted after paraffin removal and macrodissection using the Forensic kit and an iPrep system according to the manufacturer's recommendations (Invitrogen, Life Technologies SAS, Villebon sur Yvette, France). The DNA concentration was quantified by spectrophotometry (NanoDrop ND-100 instrument; Thermo Fisher Scientific, Waltham, MA, USA) and normalised to $5 \mathrm{ng} / \mu 1$.

Detection of BRAF V600 mutations. Detection of the most frequent BRAF mutations was performed by allele-specific amplification as previously described (19) with minor modifications. Two forward primers with variations in their $3^{\prime}$ nucleotides to be specific either for the wild-type (V600; AG GTGATTTTGGTCTAGCTACAGT) or the mutated variant (600E; AgGtGATTTTGGTCTAGCTACAGA), and one common reverse primer (AS; ATGGATCCAGACAACTGT
TCAAAC) were designed. The sequence-specific forward and reverse primers were then combined in 'Primer mix V' (primers V600 and AS) and 'Primer mix E' (primers 600E and AS). The amplification conditions were optimised for the RotorGene 3000 instrument (Qiagen, Courtaboeuf, Ozyme, Saint Quentin en Yvelines, France). For each sample, the $\mathrm{Ct}$ value was determined for the V600 (control) PCR and for the 600E (mutation-specific) PCR, and the difference was calculated $(\Delta \mathrm{Ct})$. The lower was the amount of mutated DNA in the sample, the higher was the $\Delta \mathrm{Ct}$ value. Serial dilutions of mutant DNA (Colo205 cell line) in a background of wt DNA allowed determination of a cut-off value of 5. Thus, samples with $\Delta \mathrm{Ct}<5$ were considered as positive for the p.V600E mutation.

This assay can detect (but not distinguish) the V600E, V600K and V600D mutations, but not the V600R mutation. Therefore, each sample was further analysed by conventional Sanger DNA sequencing using BRAF 15S (TCATAATGCT TGCTCTGATAGGA) and BRAF 15AS (GGCCAAAAATT TAATCAGTGGA) primers for both amplification and sequencing.

Assay performances. Our assay sensitivity was determined using serial dilutions of mutated DNA (Colo205 cell line) in wild-type human genomic DNA (Invitrogen). The allelespecific amplification sensitivity was $5 \%$ for the V600E mutation, and DNA sequencing allowed detection of a BRAF V600 alteration when it was present in at least $10 \%$ of cells. In light of these results and to avoid false-negative results, we considered that a minimum of $10 \%$ of tumour cells had to be present in the sample. If no alteration was found in a sample presenting $<10 \%$ of tumour cells, we concluded that the test was not contributive.

Our laboratory has been involved in external quality schemes organized in Western France in 2011 and more recently in the National External Quality Control Scheme organized by the French National Cancer Institute. We obtained the expected results for all the samples tested using our procedures.

Statistical analysis. Overall and progression-free survivals were determined for the 278 patients and the 91 primary melanomas. As mucous melanomas are currently considered apart from the other primary melanomas, they were not included in the statistical analysis focusing on primary melanomas. Clinicopathological characteristics were tested for their association with the BRAF mutation using the Fisher's exact test or Pearson's $\chi^{2}$ test. Log-rank tests and Cox models were used for survival analyses. As vemurafenib has been demonstrated to improve survival, the survival analysis was completed with a censored analysis of the patients treated with this drug at the first day of treatment. For all analyses, two-tailed $\mathrm{P}<0.05$ was set to indicate statistical significance. Statistical analyses were performed using the R2.15.1 statistical software version.

\section{Results}

Patients. A total of 367 melanoma samples from 278 patients (138 females and 140 males) were included (Fig. 1). Among the 367 samples, 91 were primary cutaneous melanomas, 


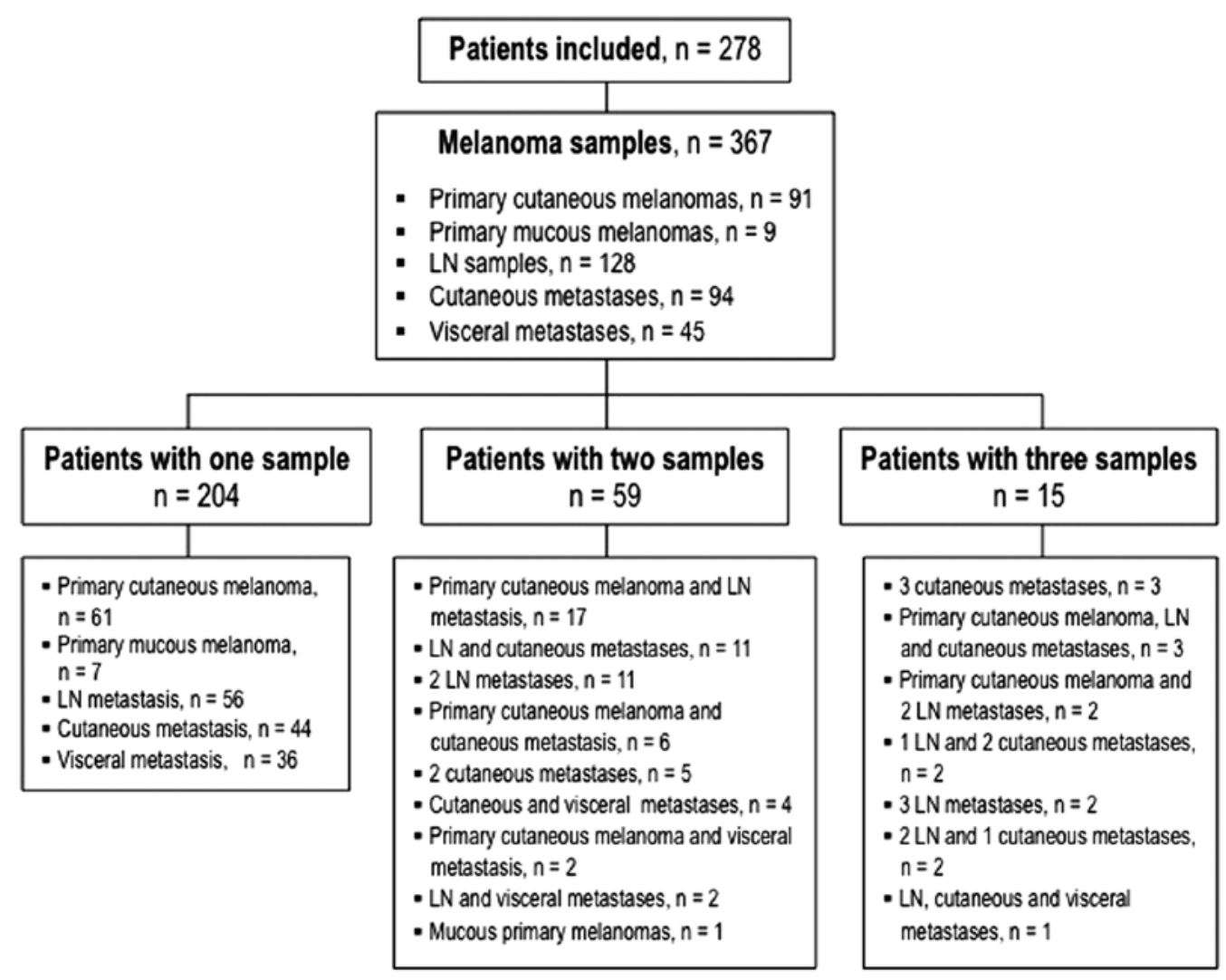

Figure 1. Patients and samples screened for their BRAF status in the present study. LN, lymph node.

Table I. Characteristics of the 278 patients tested for BRAF mutation.

\begin{tabular}{lccccc}
\hline & $\begin{array}{c}\text { BRAF-mutated } \\
\text { patients }(\mathrm{n}=114) \\
\mathrm{n}(\%)\end{array}$ & $\begin{array}{c}\text { BRAF-wild type } \\
\text { patients }(\mathrm{n}=164) \\
\mathrm{n}(\%)\end{array}$ & OR & $95 \% \mathrm{CI}$ & P-value \\
\hline $\begin{array}{l}\text { Gender } \\
\text { Female }\end{array}$ & $51(45)$ & $87(53)$ & 1.4 & $0.83-2.32$ & 0.182 \\
Male & $63(55)$ & $77(47)$ & & & $<0.001$ \\
Age (years) & & & & & $<0.001$ \\
Mean age & 55.4 & 66.5 & & & \\
$<55$ & $51(45)$ & $36(22)$ & & & \\
$\geq 55$ & $63(55)$ & $128(78)$ & & & \\
\hline
\end{tabular}

OR, odds ratio; CI, confidence interval.

9 mucous melanomas, 128 lymph node metastases, 94 cutaneous metastases and 45 visceral metastases. The BRAF mutation was found in 152 samples $(41.4 \%)$ corresponding to 114 patients $(41 \%)$. Seven samples were V600K-mutated (2\%), 2 were V600R $(0.6 \%)$ and all the others had the V600E mutation. The characteristics of these 278 patients are summarised in Table I. BRAF-mutated patients were significantly younger than the BRAF wild-type patients $(\mathrm{P}<0.001)$. No difference was noted in regard to the gender distribution between the BRAF-mutated and BRAF wild-type patients. The median follow-up of all patients was 24.8 months, and the median follow-up of patients with stage III was 14.5 months.
In the overall population (all melanoma stages), the DFS was not significantly different from the diagnosis of primary melanoma to the first recurrence between BRAF-mutated and BRAF wild-type patients $(\mathrm{P}=0.716)$ and in the multivariate analysis, no variable (gender, age and treatments) was significantly different. The OS was not significantly different between the BRAF-mutated and BRAF wild-type patients $(\mathrm{P}=0.994)$ (Fig. 2). Forty-six patients received a BRAF inhibitor, 4 received a MEK inhibitor, 31 received ipilimumab and 95 received chemotherapy. After censoring patients treated with BRAF inhibitors, the OS and DFS remained not significantly different $(\mathrm{P}=0.0502$ and $\mathrm{P}=0.679$, respectively). 
Table II. Clinical and histopathological characteristics of the 91 cutaneous primary melanoma samples tested for BRAF mutation.

\begin{tabular}{|c|c|c|c|c|c|}
\hline & $\begin{array}{c}\text { BRAF-mutated } \\
\text { samples }(n=37) \\
n(\%)\end{array}$ & $\begin{array}{c}\text { BRAF-wild-type } \\
\text { samples }(\mathrm{n}=54) \\
\mathrm{n}(\%)\end{array}$ & OR & $95 \% \mathrm{CI}$ & P-value \\
\hline \multicolumn{6}{|l|}{ Location of primary melanoma } \\
\hline Face and scalp & $4(11)$ & $15(28)$ & & & 0.0668 \\
\hline Upper extremities & $6(16)$ & $11(20.5)$ & & & 0.7855 \\
\hline Trunk & $14(38)$ & $10(18.5)$ & & & 0.053 \\
\hline Lower extremities & $13(35)$ & $18(33)$ & & & 1 \\
\hline \multicolumn{6}{|c|}{ Age at diagnosis of primary melanoma } \\
\hline Mean age (years) & 53.78 & 68.14 & & & $<0.001$ \\
\hline \multicolumn{6}{|c|}{ UV-exposed location of primary melanoma } \\
\hline Yes & $10(27)$ & $29(54)$ & 0.3 & $0.1-0.8$ & 0.0099 \\
\hline No & $27(73)$ & $25(46)$ & & & \\
\hline \multicolumn{6}{|l|}{ Ulceration } \\
\hline Absent & $19(51)$ & $18(34)$ & & & \\
\hline Present & $15(41)$ & $25(46)$ & & & 0.256 \\
\hline Unknown & $3(8)$ & $11(20)$ & & & \\
\hline \multicolumn{6}{|l|}{ Histological subtype } \\
\hline SSM & $20(54)$ & $13(24)$ & & & $0.005^{\mathrm{a}}$ \\
\hline Nodular & $9(24)$ & $15(28)$ & & & $0.63^{\mathrm{b}}$ \\
\hline Acral lentiginous & $3(8)$ & $11(20.5)$ & & & \\
\hline Lentigo maligna & $0(0)$ & $3(5.5)$ & & & \\
\hline Desmoplastic & $0(0)$ & $2(3.5)$ & & & \\
\hline Unknown & $5(14)$ & $10(18.5)$ & & & \\
\hline \multicolumn{6}{|l|}{ Breslow thickness (mm) } \\
\hline Mean thickness & 3.3 & 4.74 & & & 0.0399 \\
\hline$<1.5$ & $4(11)$ & $4(7)$ & & & \\
\hline$\geq 1.5$ & $31(84)$ & $46(86)$ & & & 0.71 \\
\hline Unknown & $2(5)$ & $4(7)$ & & & \\
\hline \multicolumn{6}{|l|}{ AJCC stage } \\
\hline I & $16(43)$ & $8(14)$ & & & \\
\hline II & $20(54)$ & $44(82.5$ & 0.2 & $0.1-0.7$ & 0.0036 \\
\hline Unknown (diagnosis on biopsy) & $1(3)$ & $2(3.5)$ & & & \\
\hline \multicolumn{6}{|l|}{ Progression } \\
\hline To stage III & $13(35)$ & $19(35)$ & & & 0.4479 \\
\hline To stage IV & $5(14)$ & $3(5.5)$ & & & \\
\hline No progression & $19(51)$ & $32(59.5)$ & & & \\
\hline
\end{tabular}

OR, odds ratio; CI, confidence interval, ${ }^{a} \mathrm{SSM}$ vs. other subtypes; ${ }^{\mathrm{b}}$ nodular vs. other subtypes.

Discordant results. Among the 74 patients with multiple BRAF analyses (59 patients with 2 samples and 15 with 3 samples), 10 had discordant results in their BRAF status (8 patients with 2 samples and 2 patients with 3 samples). Among these 10 patients, 1 had a synchronous discordant result with a lymph-node metastasis BRAF wild-type and a cutaneous metastasis V600E BRAF mutation (17).

Primary melanomas (AJCC 2009 stage I and II). A total of 91 primary melanomas were screened for their BRAF status (Table II). No patient had two primary melanomas. For three samples, the diagnosis was confirmed with a skin biopsy, with no histopathological subtype and Breslow thickness data.

Patients with a BRAF mutation were significantly younger at the time of diagnosis (mean age, 53.78 vs. 68.14 years; $\mathrm{P}<0.001)$. BRAF-mutated melanomas were significantly more often located on a non-UV exposed area $(\mathrm{P}=0.0099)$, had a thinner Breslow thickness $(\mathrm{P}=0.0399)$ and were significantly more often of SSM subtype $(\mathrm{P}=0.005)$. The location of the primary melanoma on the trunk almost reached significance for BRAF-mutated melanomas (38 vs. 18.5\%; $\mathrm{P}=0.053$ ). In addition, no significant difference was found in regards to the 


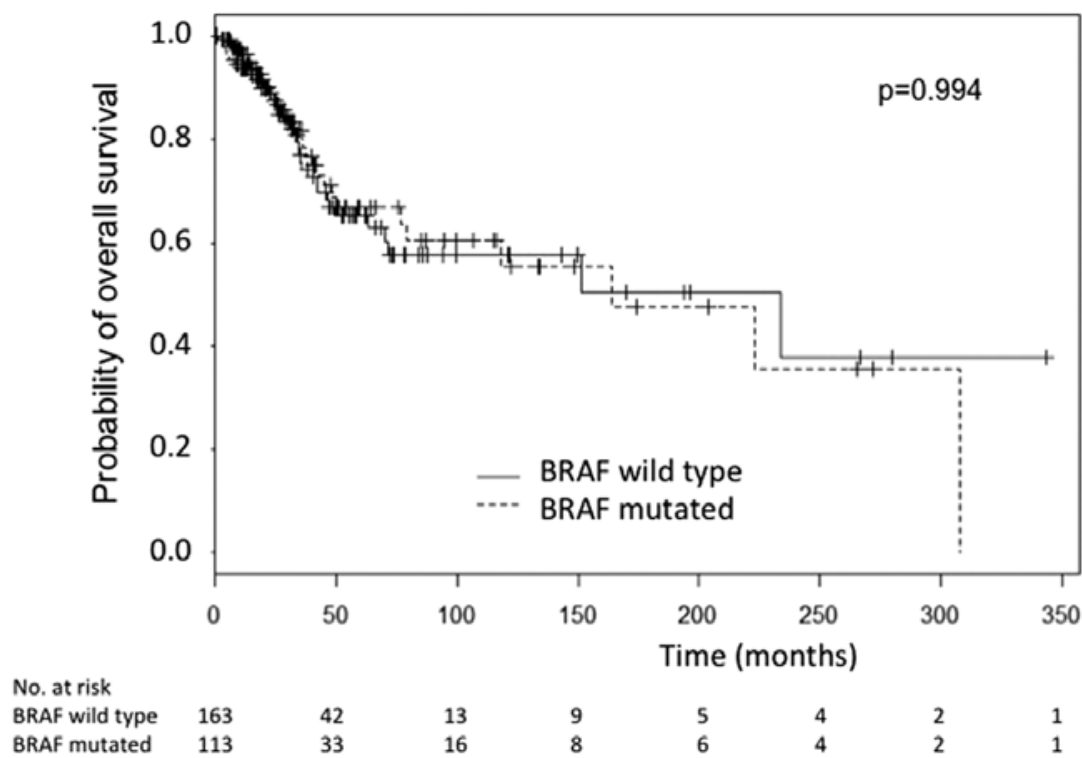

Figure 2. Overall survival of the 278 patients according to their BRAF status. For 2 patients, the date of primary melanoma was unknown, explaining 2 missing data entries in the survival analysis.

Table III. Characteristics of the 128 lymph node samples tested for BRAF mutation.

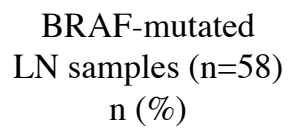

BRAF-mutated

LN samples $(\mathrm{n}=58)$

$\mathrm{n}(\%)$

\section{BRAF-wild-type \\ LN samples}

$(\mathrm{n}=70) \mathrm{n}(\%)$
OR $\quad 95 \%$ CI P-value

\begin{tabular}{|c|c|c|c|c|c|}
\hline \multicolumn{6}{|l|}{ Type of sample } \\
\hline LN biopsy & $28(48)$ & $27(39)$ & & & \\
\hline LN dissection & $29(50)$ & $42(60)$ & 0.67 & $0.3-1.4$ & 0.2833 \\
\hline NA & $1(2)$ & $1(1)$ & & & \\
\hline Number of LN invaded (for LN dissection) & 29 & 42 & & & \\
\hline 1 & $10(34)$ & $22(52)$ & & & \\
\hline$>1$ & $19(66)$ & $17(40)$ & & & 0.8028 \\
\hline NA & $0(0)$ & $3(8)$ & & & \\
\hline Capsular breaking (for LN dissection) & 29 & 42 & & & \\
\hline Yes & $17(59)$ & $22(52)$ & & & \\
\hline No & $11(38)$ & $15(36)$ & 1.05 & $0.3-3.3$ & 1 \\
\hline NA & $1(3)$ & $5(12)$ & & & \\
\hline
\end{tabular}

Type of sample

LN biopsy

LN dissection

Number of LN invaded (for LN dissection)
29

9 (66)

$17(59)$

$1(3)$
$27(39)$

$2(60)$

42

$3(8)$

42

$15(36)$

$5(12)$

LN, lymph node; NA, data not available. OR, odds ratio; CI, confidence interval.

DFS between mutated and non-mutated primary melanomas $(\mathrm{P}=0.84)$. Among the prognostic factors in the univariate analysis, the SSM subtype $(\mathrm{P}=0.15)$, thinner Breslow thickness $(\mathrm{P}<0.001)$, the absence of ulceration $(\mathrm{P}=0.0016)$ and AJCC stage I $(\mathrm{P}=0.001)$ correlated with a better DFS. The OS was not significantly different between the primary mutated and the wild-type melanomas $(\mathrm{P}=0.96)$ whereas a better OS was correlated with the absence of ulceration $(\mathrm{P}=0.012)$ and the histological subtype $(\mathrm{P}<0.001)$.

Invaded locoregional lymph nodes (AJCC 2009 stage IIIB and IIIC). One hundred and twenty-eight macroscopic lymph node samples were available (Table III). No difference in the BRAF status was noted between the number of invaded lymph nodes and the presence or absence of capsular breaking. The mean disease-free interval (DFI) between the primary melanoma and the lymph node metastasis was not different between the mutated and non-mutated patients (37.2 and 31.7 months, respectively; $\mathrm{P}=0.60)$.

Cutaneous and visceral metastases (AJCC 2009 stage IV). BRAF screening was performed in 94 cutaneous metastases and 45 visceral metastases (21 lung biopsies, 12 liver samples, 5 intestine samples, 2 bone biopsies and 1 cerebellum sample). Patients with BRAF-mutated metastases were significantly younger at diagnosis of the first metastasis $(\mathrm{P}<0.001)$. The 
BRAF mutation was found more frequently in visceral than in cutaneous metastases. The mean DFI between the primary and visceral or cutaneous metastases was not significantly different depending on the BRAF status $(\mathrm{P}=0.58)$.

\section{Discussion}

To the best of our knowledge, this is the largest study of BRAF screening in primary and metastatic melanomas with a followup until advanced stages. We included 278 European patients with 367 melanoma samples. The BRAF mutation was found in $41.4 \%$ of samples, a result similar to the proportion of BRAF mutations reported by Lee et al (7) in the largest meta-analysis performed to date.

Four retrospective cohort studies of BRAF screening in melanoma patients have previously been reported $(8-10,20)$. Our study has some critical strengths and originalities: a high number of patients, a significant proportion of patients with several samples $(74 / 278,27 \%)$, patients with melanoma of all stages (from AJCC I to IV) and a correlation with the classical prognostic factors in melanoma (including Breslow thickness and ulceration). Moreover, we determined the BRAF status for each patient using the primary melanoma itself, permitting the confirmation of a direct correlation between BRAF status and the clinicopathological type of the primary melanoma. Indeed, in other previous studies, the BRAF status used for determining this correlation was established using a metastasis sample [ $88 \%$ of the cases in the study by Long et al (10)].

BRAF-mutated patients were significantly younger at the time of diagnosis of the primary melanoma and metastasis. In the meta-analysis published in 2011, the age was not identified as a factor linked to the BRAF status but patients were separated into two groups (older or younger than 50 years of age) (7). However, many other publications have since reported a link between a younger age of patients at diagnosis of the primary melanoma and the BRAF mutation $(8,10)$ as well as in metastatic patients (20). According to our results and as suggested in a previous report by Viros et al (11), the correct 'cut-off age' appears to be 55 years. Moreover, there was no link between the occurrence of the BRAF mutation and gender, which is in accordance with other previously published results $(7,9,10)$.

According to our results, BRAF-mutated patients had the same OS and DFS than the BRAF wild-type patients. Concerning the survival analysis, two points must be considered. First of all, it is noteworthy to remember that our population was enrolled before the authorization of the temporary use of vemurafenib in France; thus, the entire population of BRAF-mutated patients included in the present study did not receive this drug. Moreover, to avoid the bias of vemurafenib effect on survival, treated patients were censored at the first day of treatment, which did not change the results of the survival analysis. The second aspect to consider is the retrospective design of the study that selected long survivors, i.e. patients with a better prognosis. We completed with a stratified analysis depending on the date of the melanoma sample (before or after January 12011 ), but the OS and DFS between BRAF-mutated patients and BRAF wild-type patients remained not significantly different.
In the present study, 91 primary melanoma samples were included to directly correlate the patient clinicopathological characteristics with the BRAF status of the primary melanoma while other more advanced melanoma samples (lymph nodes or metastases) were not considered, contrary to all the other studies published to date. Thus, the present study rules out the potential bias observed in previous studies when considering the BRAF status of metastases. We demonstrated that $13.5 \%$ of our patients had discordant results for the BRAF mutation between primary and metastatic biopsies. This could explain the differences between our results and those previously published.

The present study suggests that BRAF-mutated primary melanomas are more often located on non-sun-exposed locations (corresponding to trunk, arms, thighs and feet), are more likely of SSM subtype with a thin Breslow thickness, but are not linked to ulceration. These results are in agreement with previous publications $(7,8)$. The location of BRAF-mutated primary melanomas on the trunk almost reached significance, maybe due to a lack of statistical strength. BRAF-mutated patients were younger at the time of the first metastasis. However, the presence of a BRAF mutation in the primary melanoma was not associated with a worse survival or a more rapid progression. Five previous studies showed no link between the presence of the BRAF mutation in primary melanomas and prognosis $(9,12,13,15,21)$.

Concerning stage III, we studied the BRAF status of 128 macroscopic lymph node samples, which represents the largest study of BRAF screening on melanoma-invaded lymph nodes reported. No correlation was found between BRAF status and tumour burden represented by the number of invaded lymph nodes which is in accordance with the literature (22). Furthermore, the rapidness of lymph node relapse was not dependent of the BRAF status. This has been reported for the interval between primary melanoma and distant metastasis but never between primary melanoma and lymph node metastasis (10).

Concerning the metastatic stage, the BRAF mutation was more frequent in visceral than in cutaneous metastases. This shows the importance of verifying the BRAF status in a visceral metastasis when negative results are obtained in a cutaneous metastasis for a given patient. We confirmed that the DFI between the primary and visceral metastases (including cutaneous) was similar in mutated and non-mutated patients as already reported by Long et al (10).

Another strength of the present study was the significant proportion of patients with several samples (74/278, 27\%), allowing a comparison of the evolution of the BRAF status for a given patient with disease progression. Notably, discrepancies were found in the mutation pattern among multiple samples in 10 patients $(13.5 \%)$, similarly to what was previously observed by Colombino et al (23). We noted for the first time that this discrepancy can be observed in 2 different synchronous metastases in a same patient. Our hypothesis is based on the fact that different melanoma cell sub-clones can be present in the same tumour (24-26). This reinforces the importance of determining the BRAF status of a patient with metastatic melanoma at each step of disease progression.

In conclusion, we report here data on the BRAF mutation status in a large cohort of 278 European patients, and for the 
first time on the evolution of the BRAF status in a given patient from the primary to the metastatic stage, highlighting discrepancies according to stage. From our data, we conclude that BRAF-mutated patients are younger at the time of the primary melanoma and the first diagnosis of metastasis but these patients have the same OS than BRAF wild-type patients.

\section{Acknowledgements}

The authors gratefully acknowledge Aurore Foureau (CENGEPS network) for her helpful assistance in collecting the data and the pathologists from the Department of Pathology, Nantes University Hospital, for their contribution to the sample preparation prior to molecular testing. The Department of Biochemistry is designated and funded by the French National Cancer Institute (INCa) to perform BRAF testing of melanoma patients.

\section{References}

1. Davies H, Bignell GR, Cox C, et al: Mutations of the $B R A F$ gene in human cancer. Nature 417: 949-954, 2002.

2. Arkenau HT, Kefford R and Long GV: Targeting BRAF for patients with melanoma. Br J Cancer 104: 392-398, 2011.

3. Chapman PB, Hauschild A, Robert C, et al: Improved survival with vemurafenib in melanoma with BRAF V600E mutation. $\mathrm{N}$ Engl J Med 364: 2507-2516, 2011.

4. Hauschild A, Grob JJ, Demidov LV, et al: Dabrafenib in BRAFmutated metastatic melanoma: a multicentre, open-label, phase 3 randomised controlled trial. Lancet 380: 358-365, 2012.

5. Long GV, Trefzer U, Davies MA, et al: Dabrafenib in patients with Val600Glu or Val600Lys BRAF-mutant melanoma metastatic to the brain (BREAK-MB): a multicentre, open-label, phase 2 trial. Lancet Oncol 13: 1087-1095, 2012.

6. Flaherty KT, Infante JR, Daud A, et al: Combined BRAF and MEK inhibition in melanoma with BRAF V600 mutations. N Engl J Med 367: 1694-1703, 2012.

7. Lee JH, Choi JW and Kim YS: Frequencies of $B R A F$ and NRAS mutations are different in histological types and sites of origin of cutaneous melanoma: a meta-analysis. Br J Dermatol 164: 776-784, 2011.

8. Bauer J, Buttner P, Murali R, et al: BRAF mutations in cutaneous melanoma are independently associated with age, anatomic site of the primary tumor, and the degree of solar elastosis at the primary tumor site. Pigment Cell Melanoma Res 24: 345-351, 2011.

9. Schlaak M, Bajah A, Podewski T, et al: Assessment of clinical parameters associated with mutational status in metastatic malignant melanoma: a single-centre investigation of 141 patients. Br J Dermatol 168: 708-716, 2013.

10. Long GV, Menzies AM, Nagrial AM, et al: Prognostic and clinicopathologic associations of oncogenic $B R A F$ in metastatic melanoma. J Clin Oncol 29: 1239-1246, 2011.
11. Viros A, Fridlyand J, Bauer J, Lasithiotakis K, Garbe C, Pinkel D and Bastian BC: Improving melanoma classification by integrating genetic and morphologic features. PLoS Med 5: e120, 2008.

12. Houben R, Becker JC, Kappel A, Terheyden P, Brocker EB, Goetz R and Rapp UR: Constitutive activation of the Ras-Raf signaling pathway in metastatic melanoma is associated with poor prognosis. J Carcinog 3: 6, 2004.

13. Ellerhorst JA, Greene VR, Ekmekcioglu S, et al: Clinical correlates of $N R A S$ and $B R A F$ mutations in primary human melanoma. Clin Cancer Res 17: 229-235, 2011.

14. Safaee Ardekani G, Jafarnejad SM, Tan L, Saeedi A and Li G: The prognostic value of $B R A F$ mutation in colorectal cancer and melanoma: a systematic review and meta-analysis. PLoS One 7: e47054, 2012.

15. Nagore E, Requena C, Traves V, Guillen C, Hayward NK, Whiteman DC and Hacker E: Prognostic value of BRAF mutations in localized cutaneous melanoma. J Am Acad Dermatol: Jan 2, 2014. (Epub ahead of print). doi: 10.1016/j.jaad.2013.10.064.

16. Balch CM, Gershenwald JE, Soong SJ, et al: Final version of 2009 AJCC melanoma staging and classification. J Clin Oncol 27: 6199-6206, 2009.

17. Saint-Jean M, Quereux G, Nguyen JM, et al: Is a single BRAF wild-type test sufficient to exclude melanoma patients from vemurafenib therapy? J Invest Dermatol 134: 1468-1470, 2014.

18. Eisenhauer EA, Therasse P, Bogaerts J, et al: New response evaluation criteria in solid tumours: revised RECIST guideline (version 1.1). Eur J Cancer 45: 228-247, 2009.

19. Jarry A, Masson D, Cassagnau E, Parois S, Laboisse C and Denis MG: Real-time allele-specific amplification for sensitive detection of the BRAF mutation V600E. Mol Cell Probes 18: 349-352, 2004

20. Menzies AM, Haydu LE, Visintin L, et al: Distinguishing clinicopathologic features of patients with V600E and V600K BRAF-mutant metastatic melanoma. Clin Cancer Res 18: 3242-3249, 2012.

21. Shinozaki M, Fujimoto A, Morton DL and Hoon DS: Incidence of $B R A F$ oncogene mutation and clinical relevance for primary cutaneous melanomas. Clin Cancer Res 10: 1753-1757, 2004.

22. Moreau S, Saiag P, Aegerter P, et al: Prognostic value of $B R A F^{\mathrm{v} 600}$ mutations in melanoma patients after resection of metastatic lymph nodes. Ann Surg Oncol 19: 4314-4321, 2012.

23. Colombino M, Capone M, Lissia A, et al: BRAF/NRAS mutation frequencies among primary tumors and metastases in patients with melanoma. J Clin Oncol 30: 2522-2529, 2012.

24. Lin J, Goto Y, Murata H, Sakaizawa K, Uchiyama A, Saida T and Takata M: Polyclonality of BRAF mutations in primary melanoma and the selection of mutant alleles during progression. Br J Cancer 104: 464-468, 2011.

25. Wilmott JS, Tembe V, Howle JR, et al: Intratumoral molecular heterogeneity in a $B R A F$-mutant, BRAF inhibitor-resistant melanoma: a case illustrating the challenges for personalized medicine. Mol Cancer Ther 11: 2704-2708, 2012.

26. Yancovitz M, Litterman A, Yoon J, et al: Intra- and inter-tumor heterogeneity of $B R A F^{V 600 E}$ mutations in primary and metastatic melanoma. PLoS One 7: e29336, 2012. 